\title{
Neuronal habituation and sensitization in the reticular formation of the rat*
}

\author{
M. VIRGINIA PARKER, S. WALDEN MILLER, and PHILIP M. GROVES $\dagger$ \\ University of Colorado, Boulder, Colorado 80302
}

\begin{abstract}
Plasticity of reticular formation neuronal responses to repetitive sensory stimulation was studied in locally anesthetized paralyzed rats. Eighty-four percent of neuronal responses studied $(n=80)$ showed either decrement, increment, or increment followed by decrement in responses to repetitive sensory stimulation. Effects of stimulus parameters on neuronal plasticity were similar to effects reported for plasticity of behavioral and neuronal responses in other organisms. Spontaneous activity usually decreased as a result of stimulus repetition, although exceptions were noted. Latency from stimulus onset to first spike discharge increased during the course of stimulus repetition. Dishabituation was evident for many of the units tested, particularly for strong extra stimuli such as tail pinches. No differential location of neurons within the reticular core with respect to characteristics of plasticity was observed.
\end{abstract}

Response habituation, defined by the parameters of decrement in response to repetitive stimulation and spontaneous recovery with rest, occurs reliably in animal groups ranging from single-celled organisms to man (e.g., Humphrey, 1933). Organisms with nervous systems, however, show a wider variety of operational phenomena associated with response decrement to repetitive stimulation (see, e.g., Thompson \& Spencer, 1966; Eisenstein \& Peretz, 1973). Habituation has thus been defined by a number of operational criteria which, although as yet imprecise and lacking complete generality (see, e.g., Hinde, 1970), serve the useful function of better characterizing response decrement in organisms, and distinguishing habituation from other forms of response decrement not dependent upon alterations in the central nervous system, such as receptor adaptation and muscular fatigue (Thompson \& Spencer, 1966).

It is important, when suggesting neural systems that might mediate response hsbituation in a given class of organisms, to show that these systems have the necessary properties to account for the variety of operational phenomena which have been associated with habituation, such as response decrement, spontaneous recovery, dishabituation, and so forth. Although such demonstrations, of course, do not prove the involvement of a particular neural system, they do suggest that the properties of that system are adequate to account for the behavioral phenomena, and that it is reasonable to conclude that the system is then an appropriate

*Supported in part by Grant MH 19515 and Research Scientist Development Award K2MH 70706(PMG) from the National Institute of Mental Health. Some of the data reported here were also submitted in partial fulfillment of the requirements for the degree of Master of Arts at the University of Colorado by M. V. P. We thank Christina Cronkite for skilled of Colorado by M. V. P. We thank Christina Cronkite for skilled
technical assistance, and Jennifer Groves for preparing the illustrations.

†Address reprint requests to Philip M. Groves, Department of Psychology, University of Colorado, Boulder, Colorado 80302. candidate for theoretical consideration and further experimental investigation.

The reticular formation of the brainstem has been implicated as important for habituation of a variety of physiological and behavioral responses, at least since the classic study of habituation of the arousal response by Sharpless and Jasper (1956). Groves and Lynch (1972) recently reviewed a variety of anatomical, physiological, and behavioral evidence which seemed to support the view that the reticular formation might underlie habituation of a variety of behavioral responses. Thus, single cells in the reticular formation of the cat and rabbit demonstrate response decrement, spontaneous recovery, dishabituation, greater decrement with successive series of repetitive stimuli, and greater decrement to higher rates of stimulation (e.g., Bell et al, 1964; Segundo \& Bell, 1970; Horn \& Hill, 1966). In addition, reticular elements possess several properties which seem to characterize behavioral habituation in a variety of animals, including a lack of decrement to repetitive stimulation during sleep or barbiturate anesthesia and the "missing-stimulus effect" in which responses occur to the absence of a regularly presented stimulus following a prolonged repetitive series (Scheibel \& Scheibel, 1965).

In the experiments reported here, we confirm and extend earlier findings (Groves, Miller, \& Parker, 1972) which indicate that the characteristics of plasticity of neurons in the reticular formation of the rat are similar to those of plasticity in the reticular formation of cats and rabbits and lend additional support to the notion that the reticular formation of the brainstem is a likely candidate for the neuronal substrates of response habituation in these organisms (Sharpless \& Jasper, 1956; Groves \& Lynch, 1972). 


\section{METHOD}

Experiments were carried out on 55 adult male albino rats supplied by Horton Laboratories (Oakland, California). The rats weighed between 200 and $550 \mathrm{~g}$ at the time of the experimentation.

All surgical and experimental procedures have been described in detail elsewhere (Groves et al, 1972, 1973). Briefly, animals were anesthetized by ether inhalation, and placed in a stereotaxic instrument having blunt, hollow earbars. The calvarium was exposed and a small hole drilled over the reticular-formation target area. All points of stereotaxic and surgical contact were infiltrated thoroughly with procaine hydrochloride (Novocain) and a local anesthetic ointment. Commercial eyedrops (Visine) were applied to prevent corneal drying. Animals were then paralyzed with $2 \mathrm{mg} / \mathrm{kg}$ of tubocurarine chloride and artificially respired with a Harvard Instruments rodent respirator connected to the animal by means of polyethylene tubing and a rubber cone which fitted snugly over the snout. Heart rate was continuously monitored.

Unit activity was recorded utilizing glass-coated tungsten microelectrodes having tip diameters of from 1-7 microns, and impedances of from 0.4 to 2.5 megohms. Amplification was accomplished by means of Tektronix 122 preamplifiers and 3A9 differential amplifiers in a Tektronix 565 oscilloscope. Data were recorded on film and magnetic tape. Only single-unit activity meeting a signal-to-noise criterion of $3: 1$ or more was used for analysis.

Stimuli consisted of light flashes from a photostimulator placed approximately $15 \mathrm{in}$. in front of the $\mathrm{S}$, a variety of tactile stimuli including touching the body surface, mild footshock (1-msec pulse; 0.06-0.2 $\mathrm{mA}$ ) delivered through surface electrodes at tached to the hindfoot, blowing against the body surface, and occasionally pinching the skin or tail. Auditory stimuli were 1 -msec square wave clicks delivered through the earbars (approximately $95 \mathrm{~dB}$ intensity re: 0.0002 microbar).

A standard experimental procedure included isolation of a single-unit discharge by advancing or withdrawing the microelectrode (accomplished by means of a hydraulic microdrive which could be operated remotely), monitoring spontaneous activity for a period of several minutes, presentation of various stimuli at $2-5$ min intervals to determine the response characteristics of the single cell, and finally, repetitive stimulation at intervals of from 2 to $60 \mathrm{sec}$ for $25-200$ trials in different experiments. Specific variations in procedure are discussed where appropriate in the results section. Spontaneous recovery was used in all cases as the criterion to determine if the alterations in unit activity would be included in the sample of neurons reported here. The criterion used to define a response to sensory stimulation was an alteration in firing rate of $20 \%$ within $500 \mathrm{msec}$ following stimulation. A change in responsiveness greater than $20 \%$ to repetitive stimulus presentation was used as the criterion for habituation or sensitization

Locations of electrode tips were determined by passing current through the microelectrode, usually at the base of a microelectrode track. Lesion placements in serial sections stained with thionin were then used to determine electrode tip placements for all activity recorded in a single electrode track. Locations of unit activity were transferred to histological drawings of the appropriate brain level (DeGroot, 1959; Koenig \& Klippel, 1963; Skinner, 1971). Seven representative histological sections were chosen (corresponding to the anterior-posterior stereotaxic levels of $+1.4,+0.6,-0.1,-0.5$, $-0.75,-1.25$, and $-1.75 \mathrm{~mm}$ relative to stereotaxic zero), and the location of each neuron was transferred to the nearest representative histological section for final analysis. Only cells located within or along the borders of the mesencephalic-pontine reticular formation were included in the sample.

\section{RESULTS}

The activity of 80 single neurons, studied during repetitive stimulation, met the criteria of histological location and, where appropriate, spontaneous recovery. The response properties and characteristics of plasticity displayed by these neurons are summarized in Table 1 . Eighty-four percent of the cells studied showed some form of response plasticity to iterated sensory stimulation (habituation and/or sensitization); $16 \%$ showed no consistent alteration in response to repetitive stimulation. Notable in Table 1 is the indication that the probability of a cell displaying response alterations might be related to the extent of sensory convergence upon that cell. An average of $77 \%$ of the neurons responsive to only one modality of sensory stimulation showed plasticity of response to repetitive stimulation while an average of $94 \%$ of those responsive to two modalities displayed alterations in response to iterated sensory stimulation, and $100 \%$ of those neurons responsive to all three modalities of stimulation showed such alterations.

Figure 1 illustrates response decrement and spontaneous recovery for a reticular neuron responsive to visual stimulation. Light-flash stimuli (Grass photostimulator, intensity 16) were presented at 5-sec intervals (V 5-sec) for 30 trials. Whereas the cell

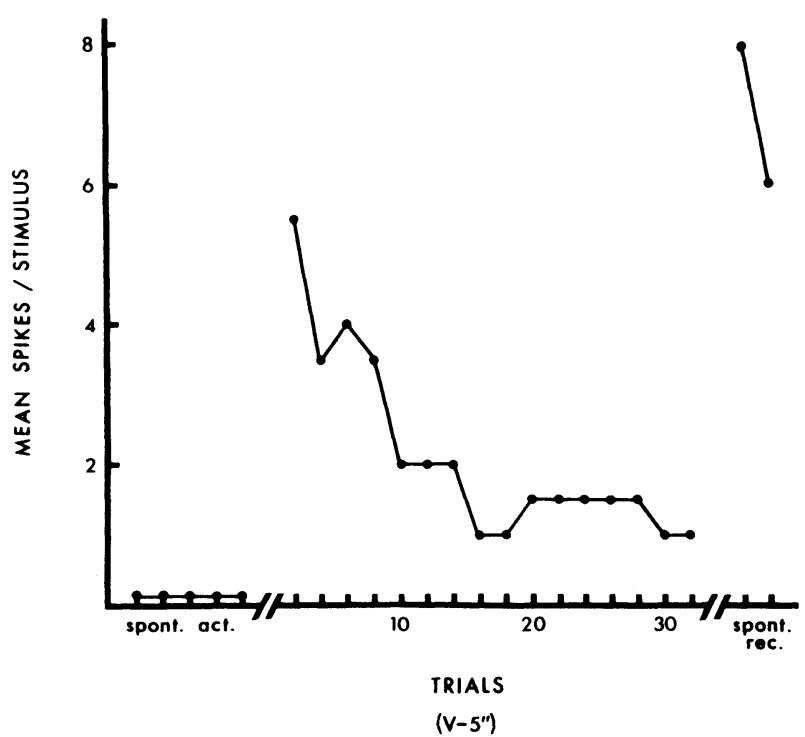

Fig. 1. Habituation and spontaneous recovery of a reticular-formation neuron responsive to visual stimulation. A response was defined as the number of spike discharges occurring within a 200-msec interval following the visual stimulus. Spontaneous activity refers to the number of spike discharges observed during a similar period of time in the absence of stimulation. Five such observations were taken randomly prior to habituation training. Thirty-two visual stimuli were presented at 5-sec intervals (V 5-sec), each point on the graph representing the mean of two consecutive responses. Following a 30-min period without stimulation, spontaneous recovery was observed. 
responded initially with 6 discharges/stimulus, this firing rate decreased to less than 2 discharges/stimulus within 15 trials. Spontaneous recovery was complete following a 30-min interval without stimulation. Typically, spontaneous recovery was complete within $10-30 \mathrm{~min}$ following habituation training for all activity recorded in our experiments.

In two cases, the response to sensory stimulation was a marked inhibition of firing. Although we encountered only two neurons during the course of these experiments that demonstrated an overall cessation of firing to the presentation of a sensory stimulus, both of these neurons displayed similar alterations in firing to repetitive sensory stimulation. The inhibitory response in both instances waned during repetitive stimulation and recovered following a period of $30 \mathrm{~min}$ without stimulation. Thus, both increases and decreases in firing rate produced by sensory stimulation may wane during habituation training procedures.

Figure 2 illustrates sensitization of activity of a neuron response to footshock stimulation $(0.2 \mathrm{~mA})$. Of our total sample of single-cell activity, $27 \%$ of the neurons showed sensitization (progressive increase in firing to repetitive stimulation) or initial sensitization followed by a progressive decrease in firing to the repetitive stimulus. Sensitization was characterized by generalization to other modalities of stimulation. An

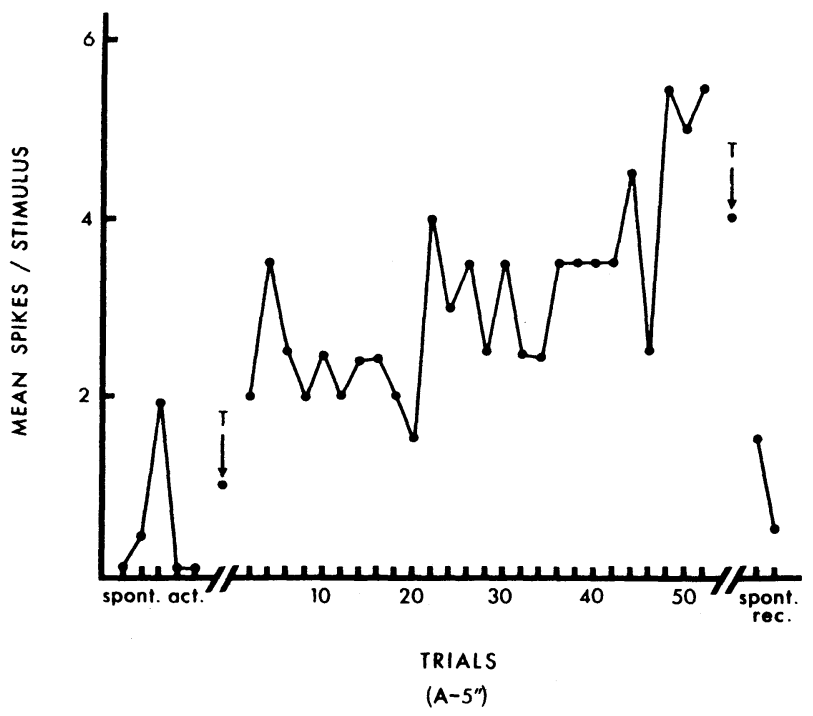

Fig. 3. Generalization of sensitization of a reticular-formation neuron responsive to auditory and tactile (footshock) stimulation. A response to auditory or tactile stimulation was defined by the number of spike discharges occurring within $200 \mathrm{msec}$ following stimulation. Spontaneous activity refers to five random observations of activity during a similar period of time in the absence of stimulation. Initially, the response to tactile stimulation (T) was quite small. Following the presentation of 52 auditory stimuli, which produced marked sensitization, the response to tactile stimulation was enhanced. Following a 30-min period without stimulation, spontaneous recovery was observed.

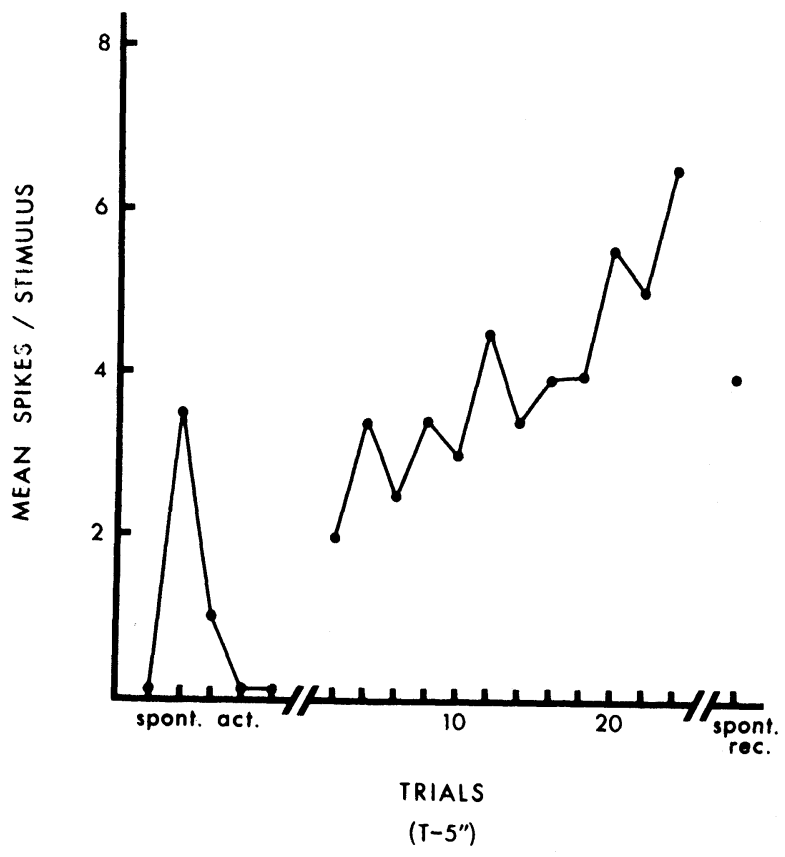

Fig. 2. Sensitization and spontaneous recovery of a reticular-formation neuron responsive to footshock stimulation (1-msec pulse; $0.2 \mathrm{~mA})$. A response was defined by the number of discharges occurring within a 200 -msec period following the tactile stimulus. Spontaneous activity refers to the number of spike discharges observed during a similar period of time in the absence of stimulation. Five such observations were taken randomly prior to habituation training. Twenty-four tactile stimuli were delivered at a rate of $1 / 5 \mathrm{sec}$ (T 5-sec). Following a 30 -min period without stimulation, spontaneous recovery was observed.

example of this is shown in Fig. 3. In this example, a neuron initially responsive only to auditory stimulation was subjected to repetitive presentation of the auditory stimulus at 5-sec interstimulus intervals (A 5-sec). The response to footshock stimulation $(0.2 \mathrm{~mA})$ prior to repetitive stimulation was not different from spontaneous firing rate. However, at the end of the repetitive auditory stimulation, which produced sensitization, the response to tactile stimulation was markedly enhanced. Habituation training in several cases produced a similar effect. A cell responsive to only one modality prior to habituation training might respond to a stimulus of a different modality following marked habituation to the initially effective stimulus. However, decrement in response was never seen to generalize to a modality of stimulation different from that used for habituation training. For neurons responsive to several modalities of stimulation, habituation to one modality never produced a consistent decrease in response to a subsequent test stimulus of a different modality. In a similar vein, cells responsive to more than one modality of stimulation could behave quite differently to repetitive stimulation, depending upon which modality was used for habituation training. Thus, a neuron might show sensitization to one modality but habituation to another. 
An example of habituation to two different rates of stimulus presentation for a neuron responsive to tactile stimulation is shown in Fig. 4. Tactile stimuli (footshock at $0.2 \mathrm{~mA}$ ) were delivered first at a rate of $1 / 5$ sec. A 30-min period without stimulation followed, and then footshock stimuli were again delivered at a slower rate $(1 / 10 \mathrm{sec})$. Note that habituation was more pronounced in the same number of trials at the faster rate of stimulus presentation. In some cases, response decrement reached the same level but at a slower rate. In other cases, the differences were negligible. The greatest overall differences occurred, as expected, with extremes in rates of presentation (e.g., 5 -sec interstimulus intervals vs 20 -sec interstimulus intervals).

In four animals, the effects of different stimulus intensities on habituation were determined. An example is shown in Fig. 5. In this case, a weak tactile stimulus (1-msec, 0.06-mA pulse delivered to surface electrodes on the hindpaw) was presented for 24 trials at a rate of $1 / 5 \mathrm{sec}$. Following a 30 -min period without stimulation, a stronger tactile stimulus was presented $(0.2 \mathrm{~mA})$ for a similar number of trials. As shown in Fig. 5, habituation to the weaker stimulus was somewhat more rapid and

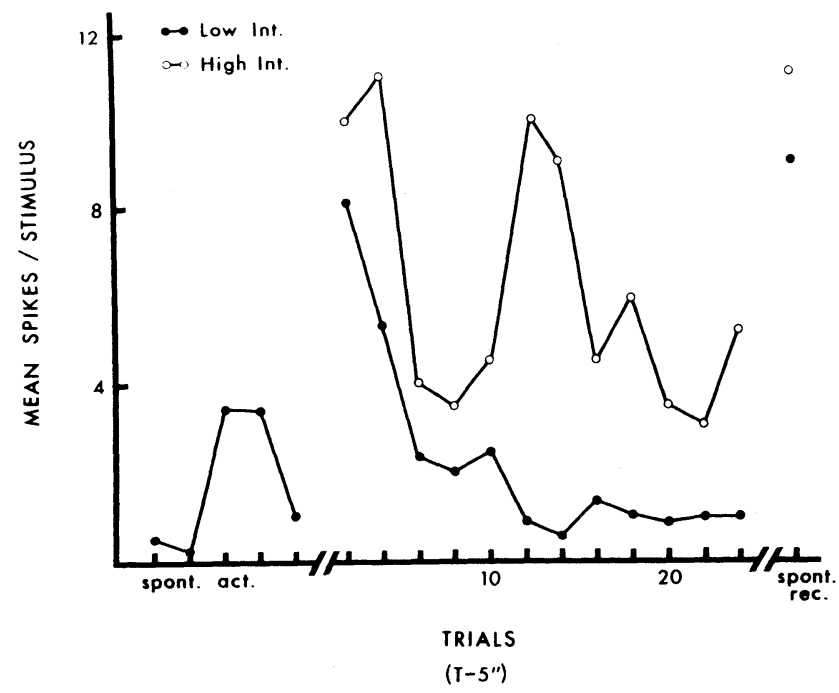

Fig. 5. Habituation of a reticular neuron response to footshock stimulation of two different stimulus intensities. A response was defined by the number of spike discharges occurring within $500 \mathrm{msec}$ following presentation of the stimulus, each point above being the mean of two consecutive responses. Spontaneous activity refers to the number of spike discharges occurring during a similar period of time in the absence of stimulation. Five such observations were taken randomly prior to habituation training. Twenty-four low-intensity tactile stimuli $(0.06 \mathrm{~mA})$ were delivered at 5 -sec intervals, a 30-min period followed during which no stimulation occurred, and then 24 higher-intensity stimuli $(0.2 \mathrm{~mA})$ were delivered through the surface electrodes attached to the hindfoot. Habituation was more rapid and more pronounced to the low-intensity stimulus. Following a $30-\mathrm{min}$ period without stimulation, spontaneous recovery was observed.

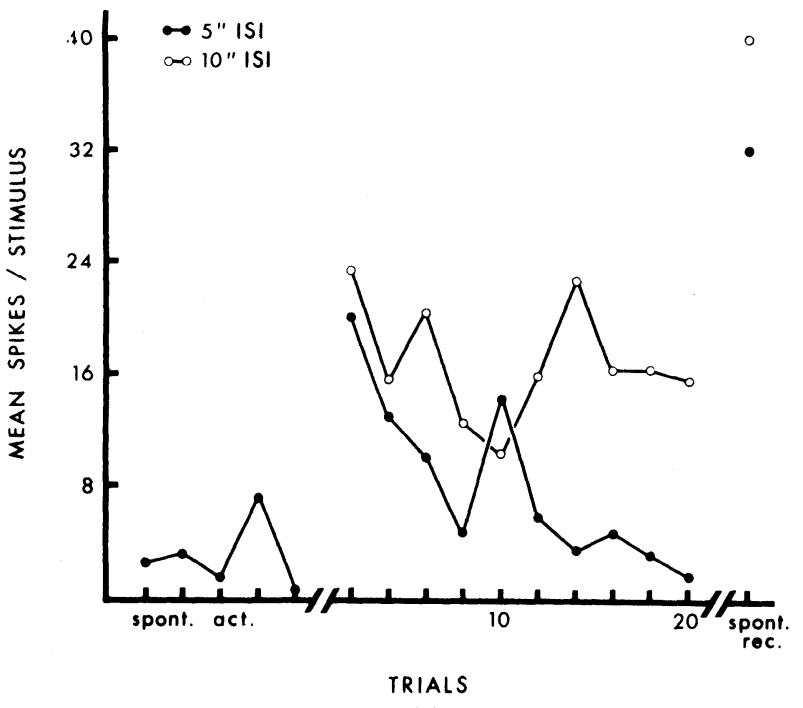

(T)

Fig. 4. Habituation of a reticular neuron responsive to footshock stimulation for two different frequencies of stimulus presentation. A response was defined by the number of spike discharges occurring within a 500-msec period following the tactile stimulus, with each point on the graph representing the mean of two consecutive responses. Spontaneous activity refers to the number of spike discharges occurring in a similar period of time in the absence of stimulation. Five such observations were taken randomly prior to habituation training. A series of tactile stimuli (mild footshock, $0.2 \mathrm{~mA}$ ) was delivered at a rate of $1 / 5 \mathrm{sec}, 30 \mathrm{~min}$ without stimulation followed, and the same stimuli were delivered again but at a rate of $1 / 10 \mathrm{sec}$. Response decrement was more rapid and more pronounced with the higher rate of stimulus presentation. Following a period of $30 \mathrm{~min}$ without stimulation, spontaneous recovery was observed.

responsiveness fell to lower levels compared to the more intense tactile stimulus. In one of four cases, there was no difference between rate or degree of habituation for two different intensities of stimulation. However, in all cases, the more intense series of stimuli was delivered second, adding the potentially confounding influence of repeated habituation training (see, e.g., Thompson \& Spencer, 1966).

Systematic attempts to produce dishabituation were carried out in 11 animals. Figure 6 illustrates one such experiment in which, while recording from a neuron responsive to visual and auditory stimuli, repetitive auditory stimulation produced habituation and, on several occasions, extraneous visual stimulation was presented. Dishabituation (a mean change greater than any spontaneous fluctuations) to the auditory stimulus was not evident either the first or the second time that the visual stimulus was presented. In 7 of the 11 cases tested, our routine sensory stimuli similarly failed to produce obvious dishabituation, while dishabituation was evident in the other 4 cases. In several of these cases, we tried pinching the tail of the animal and this turned out to be a very effective dishabituation stimulus, as 
shown in Fig. 7. This suggested that the intensities of our routine sensory stimuli were, perhaps, too low to consistently produce pronounced dishabituation.

The effect of habituation training on spontaneous activity was systematically explored in 15 animals. One-minute samples of spontaneous activity were taken immediately prior to and immediately following habituation training. In 13 cases, there was a decrease in spontaneous activity as a result of habituation training, while in two cases spontaneous activity was greater after habituation training. A comparison between initial rate of spontaneous activity and characteristics of plasticity was also undertaken. An analysis of variance revealed no significant differences in spontaneous activity for cells which showed habituation, sensitization, or no change in response to repetitive stimulation.

Similar comparisons were made for response latency. Response latencies, defined as the interval between stimulus onset and first spike discharge, were determined from film records of responses of 48 reticular neurons, both prior to and to the final five stimuli of habituation

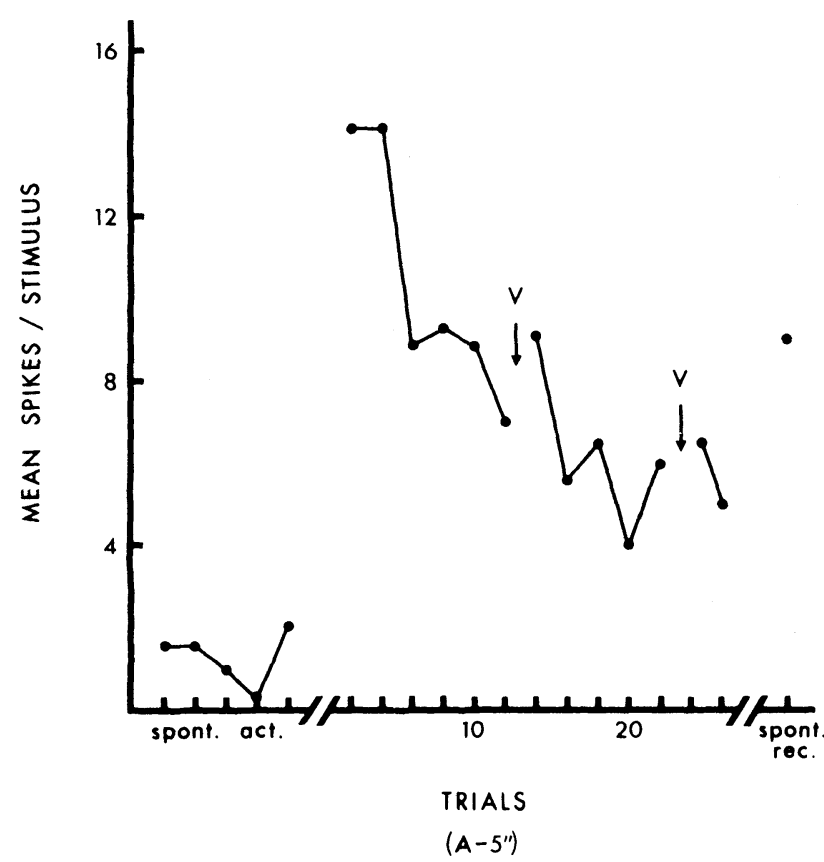

Fig. 6. An attempt to produce dishabituation by extraneous stimulation for a reticular-formation neuron responsive to auditory and visual stimulation. A response was defined by the number of spike discharges occurring within $500 \mathrm{msec}$ following the stimulus, each point above representing the mean of two consecu tive responses. Spontaneous activity refers to the number of discharges occurring during a similar period of time in the absence of stimulation. Five such observations were taken randomly prior to habituation training. Auditory stimuli were delivered at a rate of $1 / 5 \mathrm{sec}$, with an extraneous visual stimulus (V) presented twice during habituation training, as indicated by the arrows. Repetitive auditory stimulation resulted in habituation while extraneous visual stimulation had little effect. Following a 30-min rest interval, spontaneous recovery was observed.

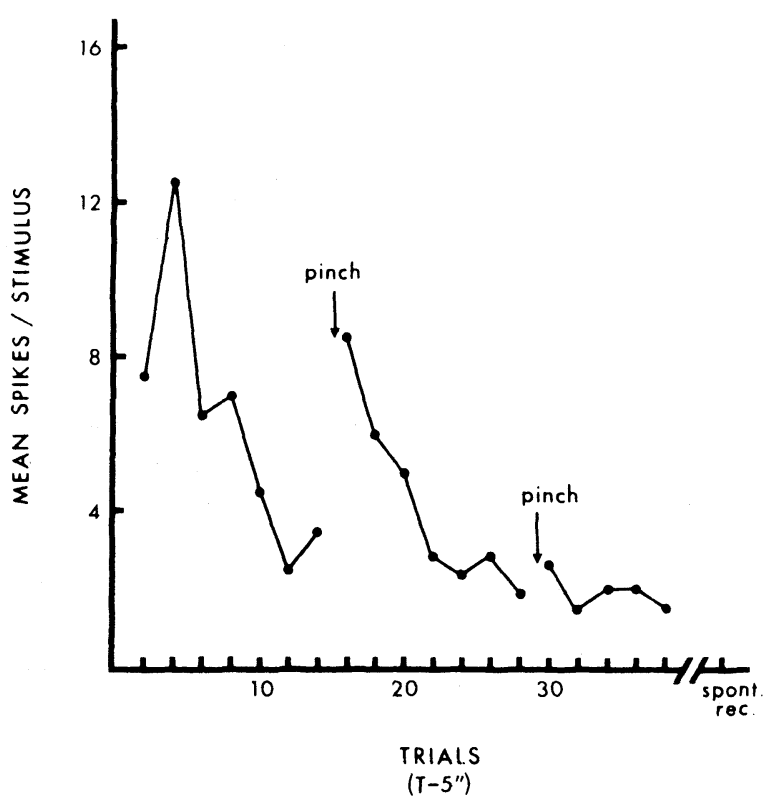

Fig. 7. Dishabituation produced by extraneous stimulation. Tactile stimuli (lightly stroking the back with a wooden probe) were delivered at a rate of $1 / 5 \mathrm{sec}$. During habituation, the tail of the rat was pinched, as indicated by the arrows, producing marked dishabituation following the first occurrence, and having substantially less effect following the second occurrence. A response in this case was defined by the number of spike discharges occurring within a 2 -sec period following stimulation. Each point represents the mean of two consecutive responses. Following a period of $30 \mathrm{~min}$ without stimulation, spontaneous recovery was observed.

training. The results indicated that average response latency was significantly longer $(t=2.26 ; \mathrm{p}<.05)$ after habituation training than prior to habituation training. The mean latency from stimulus onset to first spike discharge for these 48 observations was $61.3 \mathrm{msec}$ while the mean increase in latency as a result of habituation training was $36.7 \mathrm{msec}$. However, an analysis of variance again revealed no significant relationship between response latency and characteristics of plasticity to repetitive stimulation.

Histological verification of electrode tip placements was carried out and comparisons were made between response characteristics, plasticity, and histological location. Although marked differences in modality responsiveness do occur along the length of the reticular core (Groves et al, 1973), no differential location of units with respect to characteristics of plasticity was observed. Figure 8 illustrates the 80 electrode tip placements for the single-cell activity recorded during the course of these experiments.

\section{DISCUSSION}

The results of our studies of characteristics of reticular response attenuation to repetitive sensory stimulation are in agreement with a large body of data 

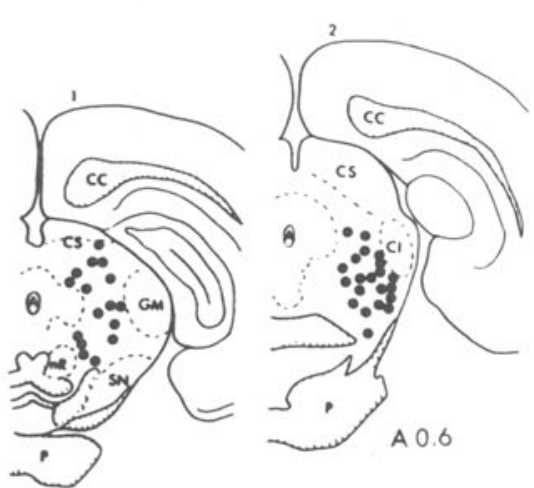

A1. 4

$$
n=16
$$

previously reported for habituation of neuronal activity in vertebrates (e.g., Segundo \& Bell, 1970), and specifically with previous reports of habituation of reticular-formation neuronal activity in the cat and rabbit (e.g., Bell et al, 1964; Horn \& Hill, 1966; Scheibel \& Scheibel, 1965). In addition, the time parameters of habituation, sensitization, and spontaneous recovery are parallel in many cases to those of habituation of behavioral responses in intact animals (e.g., Thompson \& Spencer, 1966). This is consistent with the notion that the reticular formation may underlie habituation and sensitization of behavioral responses in a variety of organisms as suggested by a number of investigators (Sharpless \& Jasper, 1956; see also Groves \& Lynch, 1972 for a review of many of these).

A number of additional points of interest emerge from analyses and comparisons of response characteristics of reticular neurons, spontaneous activity, and plasticity of response. Neuronal response decrement, for example, is often accompanied by a reduction in
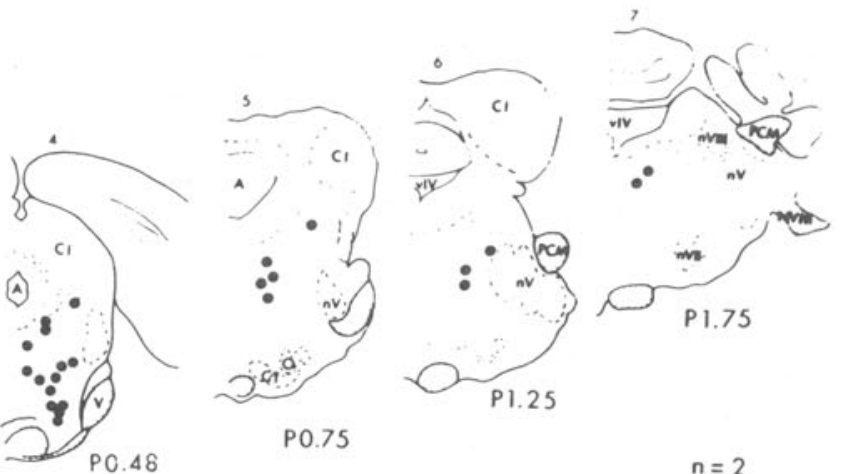

P0.75

PO. 48

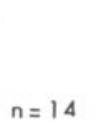

$$
n=5
$$

$n=2$

$\mathrm{n}=14$

Fig. 8. Electrode tip placements within or along the border of the reticular formation where neuronal activity was recorded. Coordinates of seven representative histological sections are given, with the number of placements in each section shown below each section.

spontaneous firing rate, although not invariably. This is in agreement with several previous reports of habituation of spinal interneuron activity (Groves, DeMarco, \& Thompson, 1969; Groves \& Thompson, 1970). However, Wickelgren (1967) and Bell et al (1964) have shown that habituation of neuronal activity can occur without significant alterations in spontaneous firing rate. As noted by others (e.g., Horn, 1970), while a consistent decrease in spontaneous activity accompanying habituation of neuronal activity might effectively support arguments that habituation occurs as a result of hyperpolarization of membranes of habituating neurons, the fact that habituation can occur without alterations in spontaneous activity precludes immediate adoption of such a hypothesis.

Response plasticity of reticular neuronal activity seemed to be related to the degree of sensory convergence displayed by reticular neurons (see Table 1). Further, plasticity might be characterized by habituation to one modality and sensitization to another

Table 1

\begin{tabular}{|c|c|c|c|c|c|c|c|}
\hline \multirow[b]{3}{*}{ Modality } & \multicolumn{7}{|c|}{ Number of Effective Modalities } \\
\hline & \multicolumn{3}{|c|}{1} & \multicolumn{3}{|c|}{2} & \multirow{2}{*}{$\frac{3}{\text { AVT }}$} \\
\hline & A & $\mathrm{V}$ & $\mathrm{T}$ & AV & AT & VT & \\
\hline Number of Cells Examined (80) & 12 & 9 & 32 & 4 & 11 & 2 & 10 \\
\hline Showed Plasticity (Percent) & 83 & 67 & 81 & 100 & 81 & 100 & 100 \\
\hline Habituation Only (Percent) & 67 & 56 & 56 & 50 & 45 & 50 & 70 \\
\hline Sensitization Only (Percent) & 16 & 0 & 9 & 0 & 9 & 50 & 0 \\
\hline $\begin{array}{l}\text { Both Habituation and } \\
\text { Sensitization (Percent) }\end{array}$ & 0 & 11 & 16 & 50 & 27 & 0 & 30 \\
\hline
\end{tabular}

Response and Plasticity Characteristics of Reticular Formation Neuronal Activity

Note $-A=$ auditory, $V=$ visual,$T=$ tactile 
(Groves, Miller, \& Parker, 1972). Finally, while sensitization to one modality often affects responsiveness of the same neuron to a different modality, there is no evidence that habituation of neuronal activity to one modality is characterized by generalization to different modalities for the same neuron. These observations sugggest that the neural processes associated with habituation, if they are reticular in origin, occur prior to points of convergence in the reticular formation. This could provide additional suggestive evidence that these processes are associated with parvicellular associational neurons within the reticular formation (Groves \& Lynch, 1972) and that these effects are ultimately relayed to effector neurons in the reticular formation, or at least points in the reticular network which must be considered to be further along in the chain of information arising from sensory input into the reticular core. Additionally, since responsiveness to different modalities of sensory stimulation is, to some extent, localized to different subregions of the reticular core of the brainstem (Groves et al, 1973), the processes of habituation and sensitization would also be expected to rely on these different subregions for plasticity to different sensory modalities. A recent analysis of habituation of the acoustic startle response in rats supports this view (Groves, Wilson, \& Boyle, 1974).

Finally, it is of interest that we have again confirmed that both increments and decrements in neuronal activity result from repetitive sensory stimulation, as reported previously for plasticity of neuronal and behavioral responses in a wide variety of organisms and systems including both vertebrates (Bell et al, 1964; Buchwald, Halas, \& Schramm, 1965; Buchwald \& Humphrey, 1973; Groves et al, 1972; Groves \& Thompson, 1970; Hinde, 1970; Horn \& Hill, 1966; Pearson \& Wenkstern, 1972; Segundo \& Bell, 1970; Scheibel \& Scheibel, 1965; Thompson \& Spencer, 1966) and invertebrates (e.g., Carew et al, 1971; Eisenstein \& Peretz, 1973; Lahue \& Corning, 1973; Rowell, 1970). Thus, there is widespread evidence for the view that plasticity of response to repetitive stimulation results from the interaction of both incremental and decremental processes.

\section{REFERENCES}

Bell, C., Sierra, G., Buendia, N., \& Segundo, J. P. Sensory properties of neurons in the mesencephalic reticular formation. Journal of Neurophysiology, 1964, 27, 961-987.

Buchwald, J. S., Halas, E. S., \& Schramm, S. Progressive changes in efferent unit responses to repeated cutaneous stimulation in spinal cats. Journal of Neurophysiology, 1965, 28, 200-215.
Buchwald, J. S., \& Humphrey, G. L. Än analysis of habituation in the specific sensory system. In E. Steallar and J. Sprague (Eds.), Progress in physiological psychology. Vol. 5. New York: Academic Press, in press.

Carew, T. J., Castellucci, V. F., \& Kandel, E. R. An analysis of dishabituation and sensitization of the gill-withdrawal reflex in Aplysia. International Journal of Neuroscience, 1971, 2, 79-98.

DeGroot, J. The rat forebrain in stereotaxic coordinates. Amsterdam: N. V. Noord-Hollansche Uitgevers Maatschappij, 1959.

Eisenstein, E. M., \& Peretz, B. Comparative aspects of habituation in vertebrates. In $H$. Peeke and $M$. Herz (Eds.) Habituation: Behavioral studies and physiological substrates. Vol. I. New York: Academic Press, 1973.

Groves, P. M., DeMarco, R., \& Thompson, R. F. Habituation and sensitization of spinal interneuron activity in acute spinal cat. Brain R esearch, 1969, 14, 521-525.

Groves, P. M., \& Lynch, G. Mechanisms of habituation in the brainstem. Psychological R eview, 1972, 79, 237-244.

Groves, P. M., Miller, S. W., \& Parker, M. V. Habituation and sensitization of neuronal activity in the reticular formation of the rat. Physiology \& Behavior, 1972, 8, 589-593.

Groves, P. M., Miller, S. W., Parker, M. V., \& Rebec, G. V. Organization by sensory modality in the reticular formation of the rat. Brain $R$ esearch, 1973, 54, 207-224.

Groves, P. M., \& Thompson, R. F. Habituation: A dual-process theory. Psychological R eview, 1970, 77, 419-450.

Groves, P. M., Wilson, D. J., \& Boyle, R. D. Brain stem pathways, cortical modulation, and habituation of the acoustic startle response. Behavioral Biology, 1974, 10, 391-418.

Hinde, R. A. Behavioral habituation. In G. Horn and R. A. Hinde (Eds.), Short term changes in neural activity and behaviour. Cambridge: Cambridge University Press, 1970. Pp. 3-40.

Horn, G. Changes in neuronal activity and their relationship to behaviour. In G. Horn and R. A. Hinde (Eds.), Short term changes in neural activity and behaviour. Cambridge: Cambridge University Press, 1970. Pp. 567-602.

Horn, G., \& Hill, R. M. R esponsiveness to sensory stimulation of units in the superior colliculus and subjacent tectotegmental regions in the rabbit. Experimental Neurology, 1966, 14, 199-223.

Humphrey, G. The nature of learning. New York: Harcourt Brace, 1933.

Koenig, J. F. R., \& Klippel R. A. The rat brain: A stereotaxic atlas of the forebrain and lower parts of the rat brain stem. Baltim ore: Williams \& Wilkins, 1963.

Lahue, R., \& Corning, W. Incremental and decremental processes in Limulus ganglia: Stimulus frequency and ganglion organization influences. Behavioral Biology, 1973, 8, 637-653.

Pearson, J. A., \& Wenkstern, B. Habituation and sensitization of the flexor withdrawal relfex. Brain $R$ esearch, 1972, 43, 107-118.

Rowell, C. H. R. Incremental and decremental processes in the insect central nervous system. In $G$. Horn and $R$. A. Hinde (Eds.), Short term changes in neural activity and behaviour. Cambridge: Cambridge University Press, 1970. Pp. 237-276.

Scheibel, M. E., \& Scheibel, A. B. The response of reticular units to repetitive stimuli. Archives of Italian Biologie, 1965, 103, 279-299.

Segundo, J. P., \& Bell, C. C. Habituation of single nerve cells in the vertebrate nervous system. In $G$. Horn and $R$. A. Hinde (Eds.), Short term changes in neural activity and behaviour. Cambridge: Cambridge University Press, 1970. Pp. 77-92.

Sharpless, S., \& Jasper, H. H. Habituation of the arousal reaction. Brain, 1956, 79, 655-680.

Skinner, J. E. Neuroscience: A laboratory manual. Philadelphia: Saunders, 1971.

Thompson, R. F., \& Spencer, W. A. Habituation: A model phenomena for the study of neuronal substrates of behavior. Psychological R eview, 1966, 173, 16-43.

Wickelgren, B. G. Habituation of spinal interneurons. Journal of Neurophysiology, 1967, 30, 1424-1438.

(R eceived for publication May 14, 1974; accepted May 23, 1974.) 